\title{
TUMOR TREATMENT BY DIRECT ELECTRIC CURRENT: COMPUTATION OF ELECTRIC CURRENT AND POWER DENSITY DISTRIBUTION
}

\author{
Damijan Miklavčič, ${ }^{1}$ Dejan Šmrov, ${ }^{1}$ Vojko Valenčič, ${ }^{1}$ \\ Gregor Serša, ${ }^{2}$ and Lojze Vodovnik ${ }^{1}$ \\ ${ }^{1}$ University of Ljubljana \\ Faculty of Electrical Engineering \\ Tržaška 25 \\ 1000 Ljubljana, Slovenia \\ ${ }^{2}$ Institute of Oncology \\ Zaloška 2 \\ 1000 Ljubljana, Slovenia
}

Key words. Electrotherapy; Tumor growth; Field Modeling; Finite element analysis.

\begin{abstract}
Electrotherapy by direct electric current was shown to have an antitumor effect in different animal tumor models and in clinic. Information on the current density distributions in the tumor, surrounding tissues, and its differences in the different electrode configurations used in our previous studies could be useful in future studies of electrotherapy mechanisms. Therefore, a three-dimensional, anatomically based, finite element model of the mouse with a subcutaneous tumor was built. Different types of electrotherapy, i.e., different electrode configurations, were modeled by applying appropriate boundary conditions in the grid points. The magnitude of current density in anodic/cathodic electrotherapy is $\mathbf{5 0}$ times higher in the middle and 10 times higher on the edge of the tumor when compared to the "field" electrotherapy. The results of the computation show that the effects of the electrotherapy on tumor growth, which were similar in terms of tumor growth delay in all three configurations, are not directly related to the magnitude of the electric current or power density in the tumor.
\end{abstract}

Address for correspondence: Damijan Miklavcic, University of Ljubljana, Faculty of Electrical Engineering, Trzaška 25, 1000 Ljubljana, Slovenia. 


\section{INTRODUCTION}

It has previously been shown that electrotherapy (ET) by low-level direct current is an effective, inexpensive, and minimally invasive tumor treatment modality. Tumor growth retardation has been demonstrated in different murine tumor models $(3,5,10$, $11,12,14,21,23)$ as well as in patients $(1,17,18,20,28)$. The antitumor effects in murine tumor models were obtained using various electrode configurations; i.e., electrodes were either inserted in the tumor or were placed subcutaneously outside of the tumor, so that the tumor lay between the two electrodes (15). Different possible underlying mechanisms of the observed phenomena already have been examined in previous studies. Tumor bioelectric potential was measured in anodic electrotherapy, as that was hypothesized to play the major role in observed antitumor effect (12). However, no change of bioelectric potential was observed due to electrotherapy (16). Changes in local $\mathrm{pH}$ and temperature were measured and calculated $(4,6,9,15)$; the role of different materials for electrodes was investigated $(15,22)$; and metal dissolution from the electrodes was determined (13). Extreme local changes of $\mathrm{pH}$ were predicted and observed around the electrodes $(4,6,9)$. Thus $\mathrm{pH}$ seems to mediate the cytotoxic effect in tumors when one or both electrodes are inserted in the tumor. These changes, however, do not explain the observed antitumor effect in field electrotherapy (15). It was also shown that the antitumor effect is not due to temperature rise, nor to metal deposited from the electrodes used $(13,15,22)$. In the present study, an anatomically based numerical model was constructed to determine electric current and power density distributions in electrotherapy where different electrode configurations were used, in order to have more precise knowledge of the levels of electric values in the tumor. The model was verified by in vivo measurements of voltage drop between five selected points and a reference point in "field" electrotherapy where both electrodes (anode and cathode) were placed subcutaneously outside of the tumor.

\section{MATERIALS AND METHODS}

An electric field in biological tissue, resulting from constant direct electric current, can be treated as quasi-stationary. It is described with the equations for steady electric currents in a volume conductor. If a conductor is homogenous, linear, and isotropic as regards electrical conductivity, then the electric potential distribution is governed by the Laplace equation. Two types of boundary conditions apply in this situation. A Dirichlet boundary condition is represented as a fixed scalar electric potential, i.e., applied voltage on the surface of the model. A Neumann boundary condition is represented as a first derivative of the scalar electric potential in the direction normal to the boundary surface of the model, i.e., current density flowing in/out of the model divided by the electrical conductivity of the tissue. In the case of the nonhomogeneous conductor, the interface conditions for the current density and electric field intensity vectors on the boundary surface between different conductivities must be considered. For anisotropic materials, the electric field distribution is governed by Kirchhoff's first law, which states that current is neither created nor destroyed; i.e., the total current into the region bounded by the closed surface must be exactly equal to the total current leaving the region. 


\section{Geometry Definition and Mesh Generation}

A three-dimensional, anatomically based, finite-element model of a mouse with a subcutaneous tumor was evolved using MSC/EMAS (Electro-Magnetic Analysis System) software package $(2,19)$. Geometry and finite-element mesh definition, as well as plotting of the results, were performed using an $\mathrm{MSC} / \mathrm{XL}$ software package, while matrix solving was done by MSC/EMAS (all trademarks of The MacNeal-Schwendler Corporation). The geometry of the model was based on the 14 cross-section scans of an $\mathrm{A} / \mathrm{J}$ mouse with a s.c. fibrosarcoma Sa-1 tumor on the left flank, obtained by magnetic resonance imaging (MRI). The distance between the two neighboring cross-sections in the longitudinal direction was $2.7 \mathrm{~mm}$. There were $11 \mathrm{MRI}$ scans in the abdominal and three in the thoracic parts of the body. The resulting three-dimensional geometric structure consisted of 11 different tissues (organs), i.e., skin, fat, skeletal and heart muscles, bone, connective tissue, intestine, kidney, liver, lung, and tumor. Each of these tissues (organs) formed a closed region. A mesh-generating tool in the software package used can generate finite-element mesh only in the brick- or wedge-shaped regions, i.e., geometric bodies with 5 or 6 boundary surfaces. Thus, the three-dimensional geometric structure representing the anatomy of the central part of the mouse had to be divided into such geometric bodies. Each region in the MRI scan, representing the same tissue (organ), was divided into yet more triangular and quadrilateral figures. Corresponding figures from two neighboring scans, together with lines connecting corresponding points, formed geometric bodies with 5 or 6 boundary surfaces, altogether constituting one slice of the model. The whole model consisted of 13 slices, 10 in the abdominal and three in the thoracic part of body. The geometry of the model was described with 1390 points which defined 3859 curves/lines. A total of 1379 geometric bodies were defined using those curves. All information about geometric entities, i.e., coordinates of points, definitions of curves, and geometric bodies, was arranged into a database using dBase IV software (Ashton Tate Corporation) in order to simplify further model modifications and mesh generation procedure.

The mesh-generating tool in the software package used requires divisions in three main axes to be defined for each brick or wedge body. The directions of axes are aligned with the edges of each body. The divisions in the boundary surface for each pair of neighboring bodies must be identical in the whole model. These elements were also arranged into the same database as previously mentioned to simplify changing of the finite-element mesh in the regions of interest. The finite-element model obtained was constructed from 9077 three-dimensional finite elements.

\section{Material Properties and Model Verification}

Different tissues (organs) are characterized by appropriate electrical conductivities. In general, they are represented with a conductivity tensor where conductivities in appropriate directions corresponding to the coordinate system used are presented. When the conductivity can be described in an orthogonal coordinate system (e.g., Cartesian), and the electric field and the current density are related to the same system, all nondiagonal elements of the conductivity tensor are zero. For the isotropic material, where conductivity is not direction-dependent, the conductivity tensor is reduced to scalar. The values of electrical conductivity of tissues used in the model are given in Table 1 . They were collected from the literature $(7,8,27)$. Skeletal and heart muscles 
Table 1. Conductivities of the Tissues in the Model

\begin{tabular}{lc}
\hline Tissue & Conductivity $\gamma(\mathrm{S} / \mathrm{m})$ \\
\hline Skin & 0.04 \\
Fat & 0.046 \\
Skeletal muscle & $\gamma_{x x}=0.225$ \\
& $\gamma_{y y}=0.225$ \\
& $\gamma_{z z}=0.9$ \\
Bone & 0.025 \\
Connective tissue & 0.025 \\
Intestine & 0.55 \\
Kidney & 1.01 \\
Liver & 0.333 \\
Lung & 0.07 \\
Heart muscle & $\gamma_{x x}=0.2$ \\
& $\gamma_{y y}=0.2$ \\
& $\gamma_{z z}=0.44$ \\
Tumor ${ }^{2}$ & 1.25 \\
\hline${ }^{2}$ The value was obtained based on model validation (see text for \\
details).
\end{tabular}

were modeled as anisotropic materials with a higher conductivity in the longitudinal direction (parallel to fibers). The coordinate systems for both conductivity tensors corresponded to the main axes of the model (Fig. 1). All other tissues (organs) were modeled as isotropic.

Since we could not find data on the electrical conductivity of Sa-1 tumor, this value was determined by "fitting" it to the measurement values. For this, voltage drop was measured between 5 selected points in the tumor and surrounding tissue and a reference point in the surrounding tissue during "field" ET with three different currents: $0.6,1.0$, and $1.5 \mathrm{~mA}$. Beside two electrodes used for electrotherapy, three additional measurement electrodes were used in the measurement setup based on an electrometer amplifier (Pt-Ir alloy 90\%-10\%, $0.7 \mathrm{~mm}$ diameter). The first measure-

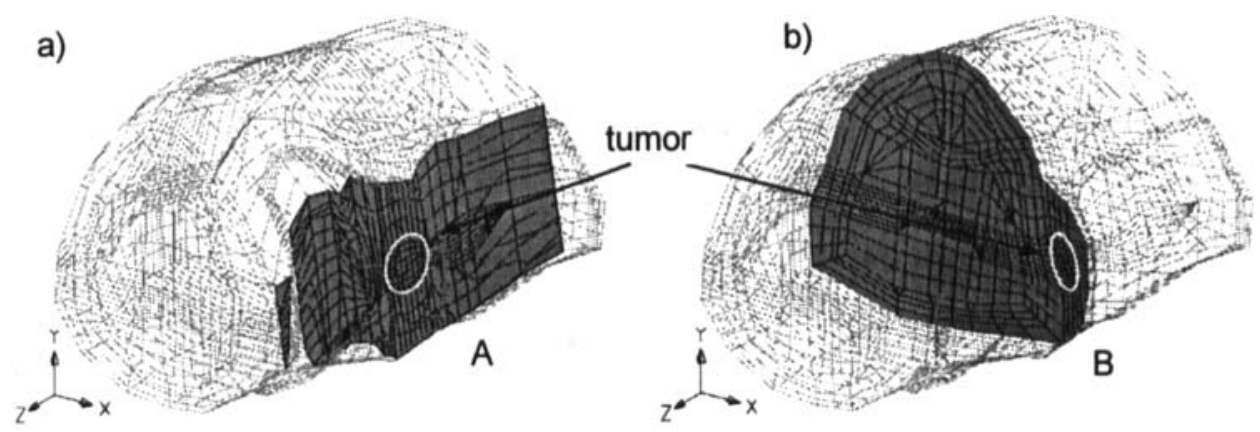

FIGURE 1. Finite element model of the mouse with cross sections A and B. The size and position of the tumor are indicated in both sections. 
ment electrode was placed s.c. parallel to the electrodes used for electrotherapy, between one electrotherapy electrode and the tumor. The second measuring electrode was placed on a manipulator enabling us to change its position. This electrode was inserted into the tumor or surrounding tissue perpendicular to the skin surface. The third reference measuring electrode was inserted s.c. in the neck of the mouse.

Since the tumor in measured animals was larger than the standard in electrotherapy electrode models, a new model was evolved with larger dimensions of the tumor. The measurements were performed with "field" ET at three currents $(0.6,1$, and 1.5 $\mathrm{mA}$ ). Therefore, three computations were performed using adequate boundary conditions. A potential at one electrode was always $0 \mathrm{~V}$, while another electrode was assigned a potential of $1.86,2.3$, and $2.82 \mathrm{~V}$ corresponding to ET with $0.6,1$, and $1.5 \mathrm{~mA}$, respectively. The voltages used were measured on the output of the DC electric stimulator (operating in the current source mode) during the experiment and reduced for electrochemical potential. The conductivity of Sa-1 tumor in the model was varied and electric potential distribution was observed to "fit" the measured values. As a result of this part of study, an electric conductivity of $1.25 \mathrm{~S} / \mathrm{m}$ was chosen for Sa-1 tumor. This value is five times greater than the conductivity of surrounding connective tissue, which also corresponds to the data in the available literature (24-26).

\section{Boundary Conditions-Electrode Configurations}

Different types of electrotherapy, i.e., three different electrode configurations, which were used in our previous experiments, were obtained by applying appropriate boundary conditions to the grid points in the corresponding regions of the finite-element model. Fixed values of scalar electric potential, i.e., Dirichlet boundary conditions, were assigned to grid points in the regions where electrodes were placed.

Since all conductors were linear, the only difference between anodic and cathodic configurations was a field reversal. Therefore, only a model of anodic electrotherapy was calculated, where the positive electrode was inserted directly into the tumor and the potential of $1.8 \mathrm{~V}$ was assigned to the corresponding grid points so that the total current flowing in/out of the model was $0.6 \mathrm{~mA}$. The negative electrode was placed subcutaneously in healthy tissue $5.4 \mathrm{~mm}$ from the tumor edge and the potential of $0 \mathrm{~V}$ was applied to the corresponding grid points. In the "field" electrotherapy both electrodes were introduced subcutaneously to either side of the tumor. Potentials of 0 and $1.2 \mathrm{~V}$ were assigned to either of the groups of the corresponding grid points representing electrodes placed $5.4 \mathrm{~mm}$ from the tumor edge on both sides of the tumor. The criteria for the voltage selection was again the total current of $0.6 \mathrm{~mA}$ flowing in/out of the model.

On the outer surface of the model, a Neumann boundary condition was applied. This boundary was considered as the interface between a conducting medium and air (assumed to be an ideal dielectric). Since the conductor (skin layer) was linear and isotropic, the usual Neumann condition was applied; i.e., the normal derivative of the electric potential on the interface between the model and surrounding air was zero.

\section{RESULTS}

Current and power density distributions were observed in two cross-sections of the model (Fig. 1). Cross-section A was chosen so that we can observe the calculation 
results in the tumor and near the electrodes (Fig. 1a). Cross-section B (Fig. 1b) lies perpendicular to $A$ and gives an insight of the electrical values in the tumor as well as information on the spread of current deeper in the body. Results of calculated current density distributions for different electrode configurations are presented in cross-sections $\mathrm{A}$ and $\mathrm{B}$.

As we have previously shown, a similar antitumor effect, resulting in tumor growth delay of approximately 2 days, was observed in three different electrode configurations (15). Electrotherapy was performed with the anode inserted centrally in the tumor and the cathode s.c. outside of the tumor (anodic ET) or vice versa (cathodic ET); or both electrodes were placed s.c. outside of the tumor so that the tumor lay between ("field" ET). Current and power density distributions were calculated for different electrode configurations in two observed cross-sections through the model.

Since the model is linear, the electric field distributins for cathodic and anodic ET are identical except for the direction of the field, which is reversed. Therefore, the results of the calculation for the anodic ET are referred to as anodic/cathodic. In Figure 2 the distributions of current density magnitude for the anodic/cathodic ET in both cross-sections are shown. The region of maximal current density is near the electrode that is inserted into the tumor and its magnitude is $85 \mu \mathrm{A} / \mathrm{mm}^{2}$. Current density magnitude falls radially to the edge of the tumor to $14.5 \mu \mathrm{A} / \mathrm{mm}^{2}$. The power density reaches its maximum near the electrode inside the tumor where its magnitude is $59 \mu \mathrm{W} / \mathrm{mm}^{3}$. At the edge of the tumor the magnitude of the power density is much lower and its value is $5 \mu \mathrm{W} / \mathrm{mm}^{3}$.

The distributions of current density magnitude for the "field" ET in both crosssections are shown in Figure 3. The region of maximal current density lies near the electrode placed toward the head of mouse and its magnitude is $27.8 \mu \mathrm{A} / \mathrm{mm}^{2}$. Inside the tumor the magnitude of current density is between 1.8 and $2.6 \mu \mathrm{A} / \mathrm{mm}^{2}$. On the right side of Figure 3 the distribution of the current density in cross-section $B$ in the direction perpendicular to the tumor is shown. A rise can be observed in the region away from the tumor toward the center of the model. This rise is a consequence of the higher conductivity of muscle tissue in that region. The power density again reaches its maximum near the electrodes where its magnitude is $7.5 \mu \mathrm{W} / \mathrm{mm}^{3}$. In the tumor the magnitude of the power density is $0.01 \mu \mathrm{W} / \mathrm{mm}^{3}$ in the middle and $0.05 \mu \mathrm{W} / \mathrm{mm}^{3}$ at the edge of the tumor tissue.
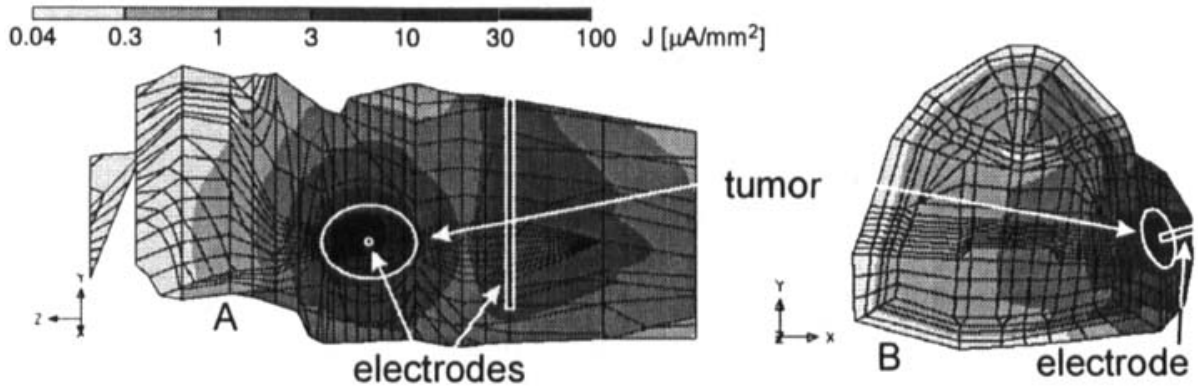

FIGURE 2. Current density distributions for anodic/cathodic electrotherapy. 

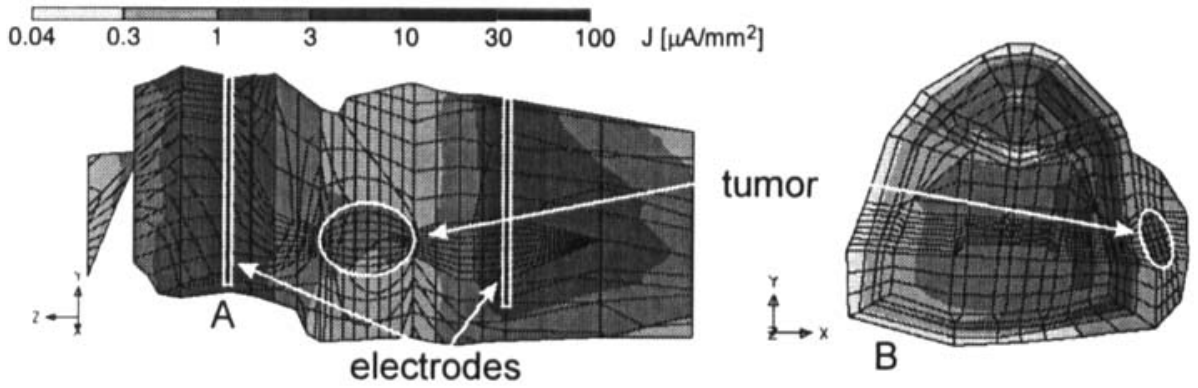

FIGURE 3. Current density distributions for "field" electrotherapy.

\section{DISCUSSION}

A three-dimensional, anatomically based model of a mouse with a subcutaneous tumor was constructed by means of the finite-element method. The model was verified by measurements of voltage drop during electrotherapy with three different magnitudes of current. Electric conductivity of $1.25 \mathrm{~S} / \mathrm{m}$ was chosen for fibrosarcoma Sa-1 subcutaneous tumor tissue, based on tumor conductivity variation study. The value chosen was five times higher than that of the surrounding connective tissue. Higher conductivity of the tumor tissue is in agreement with available data on tumor conductivity in the literature (24-26). Electrotherapy was modeled by applying appropriate houndary conditions in correspondence to different electrode configurations previously reported (anodic, cathodic, and "field" electrotherapy) (15).

From the results it can be observed that in all three types of ET, current and power densities have their maxima near electrodes, as expected. The summary data for current and power density are listed in Table 2.

The results for anodic and cathodic ET are identical except for the direction of the field, which is reversed. The field inside the tumor is very inhomogeneous. The magnitude of the current density in the middle of the tumor, i.e., near the electrode, is 6 times greater than at the edge of the tumor. The power density in the middle of the tumor is 10 times greater than at the edge of the tumor. The magnitude of the current density inside the tumor in the "field" ET is more than 10 times lower than near the

Table 2. Current ( $J$ ) and Power (p) Density in Selected Regions of the Model

\begin{tabular}{lcr}
\hline Region & Anodic/cathodic ET & "Field" ET \\
\hline J in the middle of the tumor & $85 \mu \mathrm{A} / \mathrm{mm}^{2}$ & $1.8 \mu \mathrm{A} / \mathrm{mm}^{2}$ \\
J near electrode & $85 \mu \mathrm{A} / \mathrm{mm}^{2}$ & $27.8 \mu \mathrm{A} / \mathrm{mm}^{2}$ \\
J on the edge of the tumor & $14.5 \mu \mathrm{A} / \mathrm{mm}^{2}$ & $2.6 \mu \mathrm{A} / \mathrm{mm}^{2}$ \\
p in the middle of the tumor & $59 \mu \mathrm{W} / \mathrm{mm}^{3}$ & $0.01 \mu \mathrm{W} / \mathrm{mm}^{3}$ \\
p near electrode & $59 \mu \mathrm{W} / \mathrm{mm}^{3}$ & $7.5 \mu \mathrm{W} / \mathrm{mm}^{3}$ \\
p on the edge of the tumor & $5 \mu \mathrm{W} / \mathrm{mm}^{3}$ & $0.05 \mu \mathrm{W} / \mathrm{mm}^{3}$ \\
\hline
\end{tabular}


electrode. The power density inside the tumor is more than 200 times lower than near the electrode.

The magnitude of current density in the anodic/cathodic ET compared to the "field" ET is 50 times greater in the middle and 10 times greater at the edge of the tumor. The ratio is much higher for the power density. In the middle of the tumor, the power density in the anodic/cathodic ET is 6000 times greater than in the "field" ET. On the edge of the tumor this ratio falls to 100.

The results of the computation show that the effects of the ET on tumor growth are not directly related to the magnitude of the electric field in the tumor for the parameters used in our experiments. Obtained values of current density, electric field, and power density distribution will be used for further in vitro experiments studying the effects of electric direct current on cells.

\section{ACKNOWLEDGMENTS}

We appreciate the help of Franci Demšar, D.Sc., from Institute J. Stefan, Ljubljana, who provided us with the MRI scans. This research was supported by the Ministry of Science and Technology of the Republic of Slovenia.

\section{REFERENCES}

1. Azavedo, E., Svane, G., and Nordenstroem, B.: Radiological evidence of response to electrochemical treatment of breast cancer, Clin. Radiol. 43, 84-87, 1991.

2. Brauer, J.R. and MacNeal, B.E., editors: MSC/EMAS User's Manual Version 2.5, MacNeal-Schwendler Corporation, Los Angeles, 1991.

3. Chou, C.K., McDougall, J., Ahn, C., and Vora, N.: Electrochemical treatment (ECT) of mouse and rat fibrosarcomas with direct current, Bioelectromagnetics 18(1), 14-24, 1997.

4. Cvirn, P., Rešcič J., and Miklavčič, D.: Tumor pH changes due to electrotherapyexperimental results and mathematical model, ELVEA 61, 37-42, 1994.

5. David, S.L., Absolom, D.R., Smith, C.R., Gams, J., and Herbert, M.A.: Effect of low level direct current on in vivo tumor growth in hamsters, Cancer Res. 45, 5626$5631,1985$.

6. Dodd, N.J.F., Moore, J.V., Taylor, T.V., and Zhao, S.: Preliminary evaluation of low-level direct current therapy using magnetic resonance imaging and spectroscopy, Phys. Med. IX, 285-289, 1993.

7. Geddes, L.A. and Baker, L.E.: The specific resistance of biological material-a compendium of data for the biomedical engineer and physiologist, Med. Biol. Eng. 5, 271-293, 1967.

8. Gielen, F.L.H., Wallinga-de Jonge, W., and Boon, K.L.: Electrical conductivity of skeletal muscle tissue: experimental results from different muscles in vivo, Med. Biol. Eng. Comp. 22, 569-577, 1984.

9. Griffin, D.T., Dodd, N.J.F., Zhao, S., Pullan, B.R., and Moore, J.V.: Low-level direct electrical current therapy for hepatic metastases. I. Preclinical studies on normal liver. Br. J. Cancer 72, 31-34, 1995. 
10. Habal, M.B. and Schauble, M.K.: An implantable DC power unit for control of experimental tumor growth in hamsters, Med. Instrum. 7, 305-306, 1973.

11. Heiberg, E., Nalesnik, W.J., and Janney, C.: Effects of varying potential and electrolytic dosage in direct current treatment of tumors, Acta Radiol. 32, 174-177, 1991.

12. Humphrey, C.E. and Seal, E.H.: Biophysical approach towards tumor regression in mice, Science 130, 388-390, 1959.

13. Miklavčič, D., Fajgelj, A., and Serša, G.: Tumor treatment by direct electric current: electrode material deposition, Bioelectrochem. Bioenerg. 35, 93-97, 1994.

14. Miklavčič, D., Serša, G., Vodovnik, L., Novakovič, S., Bobanovič, F., Golouh, R., and Reberšek, S.: Local treatment of murine tumors by electric direct current, Electro- Magnetobiol. 11, 109-125, 1992.

15. Miklavčič, D., Serša, G., Kryžanowski, M., Novakovič, S., Bobanovič, F., Golouh, R., and Vodovnik, L.: Tumor treatment by direct electric current-tumor temperature and $\mathrm{pH}$, electrode material and configuration, Bioelectrochem. Bioenerg. 30, 209-220, 1993.

16. Miklavčič, D., Serša, G., Novakovič, S., and Reberšek, S.: Tumor bioelectric potential and its possible exploitation for tumor growth retardation, J. Bioelectricity 9(2), 133-149, 1990.

17. Nordenström, B.: Biologically closed electric circuits: activation of vascular interstitial closed electric circuits for treatment of inoperable cancers, J. Bioelectricity 3, 137-153, 1984.

18. Nordenström, B.E.W.: Electrochemical treatment of cancer. I. Variable response to anodic and cathodic fields, Am. J. Clin. Oncol. 12, 530-536, 1989.

19. Peterson, K.H., editor: MSC/XL User's Manual Version 3A, MacNeal-Schwendler Corporation, Los Angeles, 1992.

20. Plesničar, A., Serša, G., Vodovnik, L., Jančar, J., Zaletel-Kragelj, L., and Plesničar, S.: Electric treatment of human melanoma skin lesions with low level direct electric current: an assessment of clinical experience following a preliminary study in five patients, Eur. J. Surg. (Suppl. 574), 45-49, 1994.

21. Samuelsson, L., Joensson, L., and Stahl, E.: Percutaneous treatment of pulmonary tumors by electrolysis, Radiology 23, 284-287, 1983.

22. Samuelsson, L., Jonsson, L., Lamm, I.L., Linden, C.J., and Ewers, S.B.: Electrolysis with different electrode materials and combined with irradiation for treatment of experimental rat tumors, Acta Radiol. 32, 178-181, 1990.

23. Schauble, M.K., Habal, M.B., and Gullick, H.D.: Inhibition of experimental tumor growth in hamsters by small direct currents, Arch. Pathol. Lab. Med. 101, 294-297, 1977.

24. Smith, S.R., Foster, K.R., and Wolf, G.L.: Dielectric properties of VX-2 carcinoma versus normal liver tissue, IEEE Trans. Biomed. Eng. BME-33, 522-524, 1986.

25. Surowiec, A.J., Stuchly, S.S., Barr, J.R., and Swarup, A.: Dielectric properties of breast carcinoma and the surrounding tissues, IEEE Trans. Biomed. Eng. 35, 257$263,1988$.

26. Swarup, A., Stuchly, S.S., and Surowiec, A.: Dielectric properties of mouse MCA1 fibrosarcoma at different stages of development, Bioelectromagnetics 12, 1-8, 1991. 
27. Valenčič, V., Krašna, A., Jurččč-Zlobec, B., and Berkopec, A.: Numerical calculations and comparison of electromagnetic field parameters inside biological tissue, Bioelectrochem. Bioenerg. 35, 115-119, 1994.

28. Xin, Y-L.: Advances in the treatment of malignant tumours by electrochemical therapy, Eur. J. Surg. (Suppl. 574), 31-36, 1994. 